\title{
Inter-Cell Interference Mitigation in LTE-Advanced Heterogeneous Networks by Dynamic Subframes Assignment Guaranteeing Fairness
}

\author{
Peng Li and Changrong Liao * \\ School of Electronic and Electrical Engineering, Chongqing University of Arts \\ and Sciences, Chongqing, China \\ *Corresponding Author E-mail: lchangr@163.com
}

\begin{abstract}
Dense network deployment is an important approach to meet the requirements of the future mobile communications. LTE-Advanced Heterogeneous Networks (HetNets) has been introduced by the 3rd Generation Partnership Project (3GPP) to inerease cell throughput, offload data from macro-cell and improve indoon cowerage. However, it also brings serious inter-cell interference with the co-channel deployment. Although the Almost Blank Subframes (ABS) scheme, one solution of Enhanced Inter-Cell Interference Coordination (eICIC), mitigates the interference, it results in undesizable fairness, huge difference of users' throughput in the system and even the incapability of communication. To address this problem, we introduce Dynamie Subframes Assignment Guaranteeing Fairness (DSAGF), in which the ABS paftern is set to mifigate inter-cell interference while guaranteeing fairness. As demonstrated in the simulation results, DSAGF significant decline in outage ratios of user equipments, narrowing difference of users' throughput, and improvement for@irness with inten-cell interference mitigation.
\end{abstract}

Keywords: heterogeneous networks, interference mitigation, dynamic subframes assignment, fairness

\section{Introduction}

In recent years, the users of smart phones has been increasing and data hungry application haye been used vorldwidely, which leads to the explosive increase of the wireless data iraffic. Consequently, the problem of this high data demand is extremly urgent for network operators to address with. These years witnessed the shift from 3rd Generation (3G) to Long Term Evolution (LTE) service. For example, a commercial LTE service was launched in China's in 2014. Whereas more strategies are required to meet the ongoing need of capacity. It is predicted that in 2018, the mobile data traffic will be 11 times higher than the traffic generated in 2013 [1]. To increase the network capacity, a lot of methods are suggested, including the adoption of smart antennas techniques, smarter scheduling techniques and higher order modulations. Reducing sizes through placing nore Base Stations (BSs) turned out to be the most effective method [2, 3]. The LTEAdvanced Heterogeneous Networks (HetNets) has been equipped with not only macro base station but also a lot of Low Power Nodes (LPNs), including Picocell Base Stations (Pico-BSs), Femtocell Base Stations (Femto-BSs), Relay Station (Relay) and Radio Remote Unit (RRH). A large number of LPNs are introduced in the HetNets [4]. It could reduce the distance between the wireless communication network and users; increase network capacity; shunt the load of macro cell; improve spectrum efficiency; enhance indoor coverage; develop the cell edge users' communication quality and reduce the costs of operation and maintenance. 
The purpose of deploying Pico-BS is to offload the traffic from the overload macrocell. In addition, such pico-BSs have one advantage of supplemnting coverage holes, which are existing in macrocell networks. In accordance with the specification of the Third Generation Partnership Project (3GPP), Femto-BSs, or Home evolved Node B (HeNB) should be largely placed indoors. With low costs, such BSs also can be depolyed by end users at their will, which is silimar to the operation of current Wi-Fi access points. Hence, the location and quantity of femto-BSs change while their on-off state switch, creating a dynamic environment, which is different with the working mode of macrocell networks.

As to HetNets, its main defect is causing more co-channel interference between small cells. And this exerts considerable influence when the deployment of small cells is random and dense. And dynamic interference mitigation techniques are adopted to avoid the problems of uncoordinated deployment since automatic adjustments can be made concerning the observed interference situations. The main present researches focus on the interference mitigation of data channel between small cells. Nevertheless, improving data channel performance highly depends on the efficiency of the control channels. Thereason for this is because in every subframe, the data transmission is preceded by the transmission of control channels, which, among other things, point the User Equipments (UEs) to their assigned data channels in the frequency domain.

Great attention should be paid to the corrupted reception of the control channels since it would definitely lead to incomplete transmission of the subsequent data in each subframe. Therefore effective strategies are needed to ensure the control channels performing robustly even with heavy interference. What's more, it must be noted that techniques like resource partitioning can't be applied to the control channels, yhich is different from the working manner of data channels. The impossibility of applying techniques similar to resource partitioning originates from that the control channels span the whole frequency domain.

A few techniques are developedon the purpose of protecting data and control regions synchronously for HetNets. A scheme defined in LTE-Advanced to handle the interference in macro-BSs is proposed by 15]. The scheme determines the location and number of subframes thatare to be blanked at LPNs. [6] propose a decoding-aided CRSIC receiver for LTE-Advanced systems. The key idea of the scheme is to generate a soft estimate of the useful signal using the turbo decoding scheme and cancel this from the total received signal prior to the estimation of the channels corresponding to interfering cells. In 3GPP, the Almost Blank Subframes (ABS) technique is proposed to mitigate the interference from macro-BSs to picocell users or from femto-BSs to macrocell users. Macro-BSs interfers picocell users and femto-BSs does the same thing to macrocell users. ABSs are certain predefined subframes transmitting Cell-specific Reference Signals (CRSs) yet withno data when the macro-BSs transmit subframes in the picocell case. No macro-BS interference is exerted on both control and data regions during these ABSs, therefore, cell-edge users can be scheduled with outstanding performance during them. Smiliarly, femto-BSs set ABSs and then macrocell users can be scheduled during them in the femtocell case. The interference of control channels is mitigated by the ABS echnique conveniently, however, this technique works inefficiently in uncoordinated netyorks. Under chaning interference conditions, there is no corresponding adjustments since the ABS configurations of BSs are static. In addition, BSs transmit no data during $\mathrm{ABSs}$, leading to the decrease in the utilization efficiency of the resources. And the ABS technique is incapable of reducing same-cell interference because the same frequency resources are utiized by both femto-BSs and pico-BSs users simultaneously [6]. What's more, mitigating interference between picocells and femtocells is of much more importance in dense deployments.

In the paper, based on the ABS technique, we developed a new method to solve the issues in uncoordinated networks. This method aims to reduce the interference on the control channel, thus providing better services to cell-edge users. Section 2 describes the 
system model used. A detailed description of Dynamic Subframes Assignment Ensuring Fairness (DSAGF) is given in Section 3. The simulation setup is explained in Section 4 and results are presented in Section 5. Finally, Section 6 draws conclusions.

\section{System Model}

In the paper, our attention is paid to the downlink. The system model is constituted by a conventional macrocell network which is embedded with femtocells. The DSAEF technique works at high efficiency not only for femtocells, but also for picocellular networks. In Section 4, we will give details about the exact distribution of the BSs in the simulation. We employ the standard LTE air interface [7] whose key technology is Orthogonal Frequency-Division Multiplexing (OFDM). The Resource Element (RE) is considered as the principal element in LTE. And it corresponds to one subcarrier in the frequency domain and one OFDM symbol in the time domain. A Resource Block (RB) is, a matrix of REs, which is 7 OFDM symbols $\times 12$ subcarriers. The basic unit for scheduling consists of two consecutive RBs in the time domain, and it has a time duration of one subframe. At every BSs, the timings of all subframes are the sane, and in the network all BSs are synchronous with each other. The structure of the subframe is demonstrated in Fig. 1.

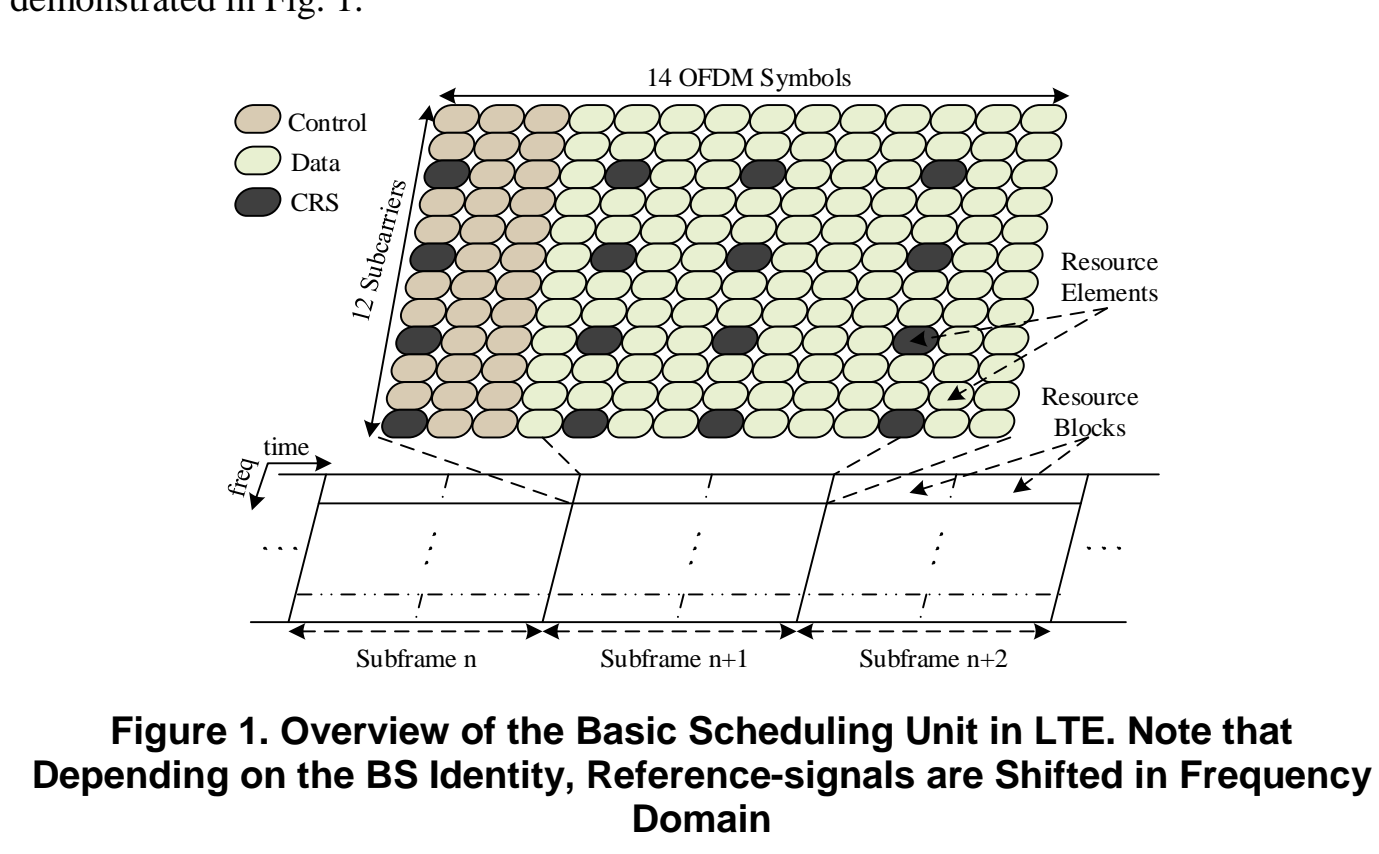

As showr in Fig. 1, each subframe is constituted by CRSs occurring periodically in time and frequency, thus facilitating channel estimation. Each subframe transmits CRSs though there is no data transmitted. Therefore, subframes during which BSs transmit no data are named ABSs. We employ a shift in the frequency domain to prevent the CRSs of neighboring cells from colliding. Hence, it is supposed that interference caused by CRSs is negligible. And more details are given in reference [8].

In LTE, UEs can differentiate between the received signals from various BSs in their vicinity with the help of CRSs. With the assistance of CRSs, signals from varieties of BSs can be differentiated by UEs in neighboring regions. The received signal strength detected by UE $u$ from BS $b$ is calculated by

$$
R_{u, b}=T^{C R S} G_{u, b}
$$

where $T^{C R S}$ is the constant CRS transmit power and $G_{u, b}$ is the channel gain which comprises the combined effect of shadowing and path loss between BS $b$ and UE $u$. 
Depending on the Reference Signal Received Powers (RSRPs), a measure report records information containing the identities of the most dominant BSs in adjacent areas [9]. And then the report is sent by the UE to its serving BS.

Nevertheless, a UE is merely capable of measuring the received power from some BSs in its vicinity. Consequently, a UE can only send its serving BS limited RSRP measurements. In addition, a UE is competent to calculate a wideband Signal to Interference plus Noise Ratio (SINR) during a subframe through the reference signals.

Finally, we assume that each BS sends the subframe pattern in this system model, that is, some subframes transmit data while some are set as ABS to its neighboring BSs since the BSs cause high interference. The subframe pattern isn't specified explicitly in the standards; however, it can be deployed so long as some minor changes are made on the signals pre-defined in specification [10]. In the following part, we will explain the usage of these signals in details.

\section{Dynamic Subframes Assignment Guaranteeing Fairness}

To further explain the working process of the DSAGF scheme, an example is hown in Fig. 2, and in the figure, there are two femto-BSs serving two UÊs along with the macroBS serving three UEs. The arrows refer to the high interference And the control channels can be decoded correctly only on the condition of such interference being mitigated. Apparently, there's always a cell edge UE needing protection in each cell. From the above, with the DSAGF, the protection can be achieved only when no same subframes is used by interfering neighbors.

In order to achieve this, a subframe pattern indicating the active and idle subframes is defined for each BS. To achieve this, we should defne a subframe for each BS, which indicates the idle and active subframes. For exanple, in Fig. 2, the subframe pattern of BSs consists of four subframes. Wo divide the overall bandwidth into four subbands, similarly, the DSAGF separates the time into four parts yet with the purpose of protecting the control channels. From the Fig. 2, we can see that data is transmitted to cell-edge user by femto-BS1 during subirame $s f_{0}$. To protect this user, femto-BS2 should transmit nothing during $s f_{\text {In }}$ another word femto-BS1 blocks $s f_{1}$ at femto-BS2. Therefore, we call $s f_{1}$ the Reservation Subframe (RS) of femto-BS1 because interfering BSs in its vicinity have to emain idles during this subframe. In the purpose of blocking a subframe at interfering BSs nearby, T/S necessary for a BS to send a so-called RS indicator to the BSs in its vicinty, which are possible to cause interference. Once the message is received by a BS, the BS sets the marked subframe as an ABS and transmits no data during the predefined subframe. Consequently, it is reduced that the interference caused to its neighboring cells. Similarly, the $s f_{1}$ should be set as ABS by femto-BS1 during the RS of femto-BS2, and so forth.

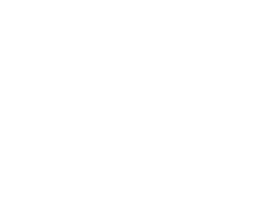




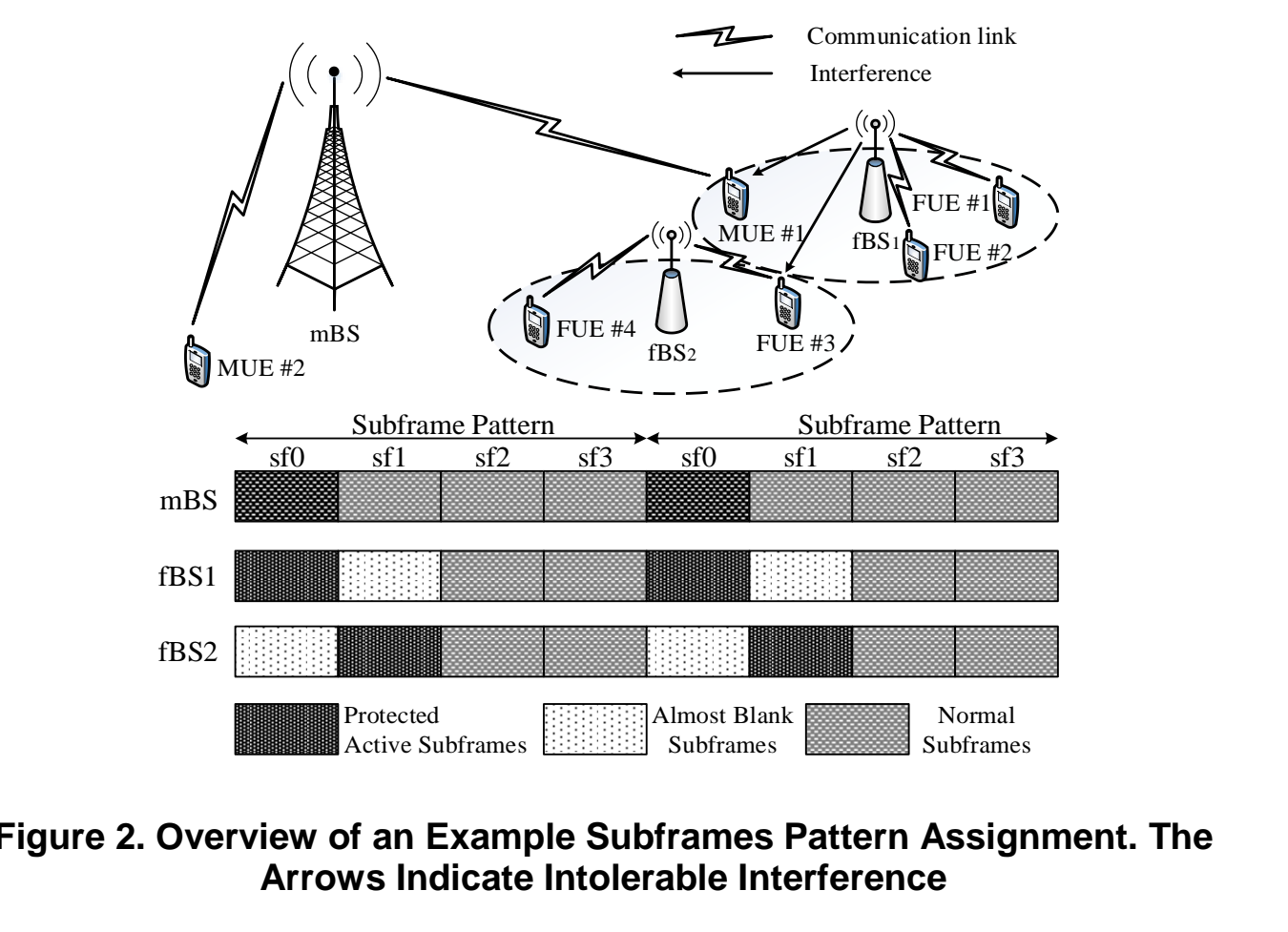

However, in terms of the macro cell, a macro-UE is trapped out of the restrictions of the femto-BS2. Obviously, with no coordination, the peighboring femtocell necessarily causes high control channel interferenee to the macro-UE. To prevent the undesirable situation, no data is transmitted to the trapped macro-UE, $M_{1}$ during $s f_{1}$ when femto-BS1 causes no interference to $s f_{0}$. In heterogeneous networks, macro-BSs are prior to femtoBSs which means that they can block subframes at femto-BSs. Nevertheless, femto-BSs is incapable of constraining macro-BSs' subframes. Nevertheless, femto-BSs can only exchange information with the neighboring BSs. We can't implement the same procedure mentioned above, which would cause numerous femto and macro interferers, further resulting in limited subframes vallable to transmit data. Finally, a great many users per cell can't be supported, which is not a desirable situation. Besides, more users are served by macro-BSs than by temo-BSs. As a result, the resource available to users served by femto-BSs is more than the resource available to per macro user.

The remaining sbuframes, including $s f_{2}$ and $s f_{3}$ of femto-BS1, are neither blocked by other BSs nor sea as reserved. These subframes can be used for relative cell-center users without limits and protection privilege. It's the primary advantage of the DSAGF. In the scheme, BS are able to protect their cell-edge users adopting RSs, meanwhile, they can use remain ng subframes on condition that they cause no or relatively low interference to their neighbors. Hence, the resource utilization finally improves for such flexibility.

The subframe patterns are updated on an event triggered, meaning that subframe pattern should be changed by their BSs when changes in the interference environment appear. BSs update their subframe patterns in a sporadic manner at the same time. The period time of updating the subframe pattern once and that of alleged time slot are the same. In this situation, a time slot is comprised of many subframe patterns. Between the beginning two time slots, the subframe patterns of BSs remain unchanged. It is only at the beginning of the time slots that BSs update their subframe pattern according to the feedback from BSs and UEs. 


\subsection{Required Measurement and Signaling}

To protect its cell-edge users, one subframe must be designated as a RS by each BS in DSAGF. To meet with this, a BS requires RS indicator and RSRP and Channel Quality Indicator (CQI) measurements, which are feedback from neighboring BSs and from its UEs respectively. The purpose of the feedback is to update the RS assignment and to identify the interfering neighbors.

It impossible for a BS to know it's all interfering neighbors since BSs are randomly deployed. To address the problem, BSs collect the measurements from UEs, which are updated periodically. We assume that all BSs remain active during the same subframes. The SINR experienced by UE $u$ served by BS $b$ is calculated as

$$
\gamma_{u}=\frac{R_{u, b}}{\sum_{m \in M_{u}} R_{u, m}+\sum_{f \in F_{u}} R_{u, \mathrm{f}}+\eta}
$$

where $M_{u}$ is the set of interfering macro-BSs, $F_{u}$ accounts for femto-BSs, and $\eta$ is thermal noise. To meet the requirement of decoding the control channels, The SINR of the control region, $\gamma_{u}$, should be no lower than the minimum SINR, $\gamma_{\text {min }}$ In other cases, we should increase $\gamma_{u}$ through removing interfering femto-BS, In other words, $\gamma_{u}$ improves when the dominant femto-BS interferen transmits nothing cluring the given subframe. Via imposing this limitation on macro-BSs, we can achieve the same effect. However, it is not allowed as the macro layer is superior to the femto layer. We have to remove the dominant interferer successively And the process will not stop unless $\gamma_{u} \geq \gamma_{\min }$ or all interfering femto-BSs are removed. The set of removed femto-BSs forms the interfering BSs of UE $u$. The femte-BSs should send ho data during the subframe that UE $u$ receives data, thus $u$ may acheve an SINR of above $\gamma_{\min }$ to decode the transmitted signals.

With the knowledge of the set of interfering femto-BSs, BS $b$ can inform the BSs of UE $u$ the potential interfering BSs on UE $u$ via a RS indicator which could be implemented as a bitmap By this means, BS $b$ keeps the interfering BSs from using the subframes which are set as RS. In the case of BS $b$ serving multiple UEs, the BS $b$ must carry out the same process for all OEs because each UE has a unique set of interfering BSs depending on.

While updating the RS must make sure that a BS has the least impact on the network. Therefore, in DSAGF, a BS classifies the subframes depending on their availability for data ransmission. A subframe can be defined as available for a user only when the user experiences an SINR above the $\gamma_{\min }$ during that subframe. This is because the user can decode the data received only in this situation. As a result, the availability of a subframe In a cell is nothing else than the number of users that experiences an SINR higher than $\mathcal{X}_{\min }$. The system sets the subframe with highest availability as the RS. And then a serving BS only has to protect fewer users via sending a RS indicator, thus decreasing the effect on the network. To obtain a subframe's availability, we utilize the wideband CQI measurements from the UEs. Note here the availability of a subframe becomes 0 when a BS receives a RS indicator which markes that subframe. By this means, we can figure out whether the subframe can be used by the BS during the next time slot. The availability of a subframe $s f$ calculated by BS $b$ as

$$
A_{s f, b}=\left\{\begin{array}{cl}
\sum_{u \in U_{b}} 1\left(\gamma_{u}^{s f} \geq \gamma_{t h}\right) & \text { if } s f \text { is not blocked } \\
0 & \text { if } s f \text { is blocked }
\end{array}\right.
$$


where $1(\cdot)$ is a conditional binary function whose output is 1 if its argument holds true and 0 otherwise. In the formula, $\gamma_{u}^{s f}$ accounts for the wideband SINR measured during the subframe $s f$ by UE $u$. $U_{b}$ stands for the set of all UEs served by BS $b$.

\subsection{Fairness Constraint}

Simply maximizing the throughput of the system will lead to serious unfairness. Some users who can't be allocated resources to, are in a completely outage state. It is necessary to suppress the inter-cell interference in the HetNets while guaranteeing the fairness of communication [11], [12]. Then the goal of optimization is no longer maximizing the number of users above the SINR threshold in the network. It should be maximizing the number of users above the SINR threshold with the prerequisite of guaranteeing certain fairness. The problem is transformed into an optimization problem with fairness constraints. $F$ is the index of measuring fairness. $F$ is calculated by

$$
F=\frac{1}{2 n^{2} E} \sum_{u=1}^{n} \sum_{v=1}^{n}\left|C_{u}-C_{v}\right|
$$

where $n$ is the number of UEs in wireless system. E is the expectation of users' throughput. $C_{u}, C_{v}$ are representing the throughput of $u$ and $v$ users respectively. Fairness index $F$ ranges from 0 to 1 . When $F$ is 0 , it means absolute fairness, which means the throughput of all users are equal; when $F$ is 1 , it means absolute unfairness. The throughput of users is calculated by equation (5) via usiggthe SINR of users.

$$
C_{u}=10 * \log _{2}(1+\times)
$$

Optimization problem with fairness constraint can be solved as follows

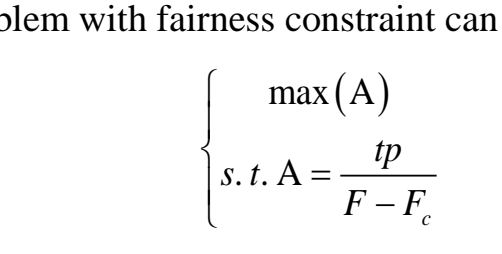

where $\mathrm{A}$ is availability of a sabframe under a current $\mathrm{ABS}$ pattern, $\mathrm{A}=\sum \mathrm{A}_{s f, b}, F$ indicates the fairness index in current $\mathrm{ABS}$ pattern, and $F_{c}$ is target fairness index.

\section{Simulation Setup}

There are seyen macro-BSs serving the whole simulation scenario. Being deployed randomly over the simulation area, Macro-UEs are served by the macro-BS, where they receive the highest power. We simulate the clustered distribution of femtocells via the employnent of the 3 GPP $5 \times 5$ grid structure [9]. The $5 \times 5$ grid stands for a square building which is composed of 25 ordered square-shaped apartments. Each apartment is equipped with a femto-BS. Each femto-BS has a closed-access policy so that every femtoBS exclusively serves a fixed set of UEs. Full-buffer transmission means all available data resources during all active subframes are assigned by macro or femto-BSs to their served UEs. In addition, each snapshot of the simulator continues for 10 time slots. The duration of each time slot equals the duration of one subframe pattern in which four subframes constitute a subframe pattern. Accordingly, 40 subframes compose a snapshot, during which it is assumed that positions and shadowing values of BSs and UEs stay the same. At the end of the 10th time slot, the statistics including SINR are calculated through adopting last four subframes, that is, the last subframe pattern. The simulation parameters are summarized in Table 1 and are in accordance with LTE specifications [13]. 
Table 1. Simulation Parameters

\begin{tabular}{cc}
\hline \hline Parameter & Value \\
\hline System bandwidth & $20 \mathrm{MHz}$ \\
Macro-BS Tx power & $46 \mathrm{dBm}$ \\
Femto-BS Tx power & $20 \mathrm{dBm}$ \\
Macro-UEs per cell & 10 \\
$5 \times 5$ grids per cell & 1 \\
Apartment dimensions & $10 \mathrm{~m} \times 10 \mathrm{~m}$ \\
Femto-BS activation probability & 0.4 \\
Femto-UES per active femto-BS & variable between 1 and 4 \\
Macro-BS shadowing std. dev. & $8 \mathrm{~dB}$ \\
Femto-BS Shadowing std. dev. & $10 \mathrm{~dB}$ \\
Fading model & No fast fading \\
Number of Tx antennas & 2 \\
Number of Rx antennas & 2 \\
\hline \hline
\end{tabular}

In LTE, $-6 \mathrm{~dB}$ or so is the minimum required SINR for decoding the control channels [14]. Hence, $-6 \mathrm{~dB}$ is set as $\gamma_{\min }$. RBs are allocated to the users during the subframe in which the user undergoes highest wideband SINR. Consequently, the UEs areassumed to go into outage when their wideband SINRs are no higher than $\gamma_{\min }$ during all subframes. In addition, 8 is the maximum number of RSRP reports send by a UE [ 5]. That is to say, the UE feeds back the reports from its own serving BS and the seven nost dominant neighboring BSs.

\section{Simulation Results}

As shown in Fig. 3, the red solid line is representing the Cumulative Distribution Function (CDF) of SINR of femto-UES without DSAGF scheme. In this case, the proportion of UEs whose SINR above the SINR threshold -6dB is relatively low. The black dotted line is representing the CDF of SINR of femto-UEs with DSAGF scheme. Adopting the DSAGF scheme the percentage of users above the SINR threshold significantly increased.

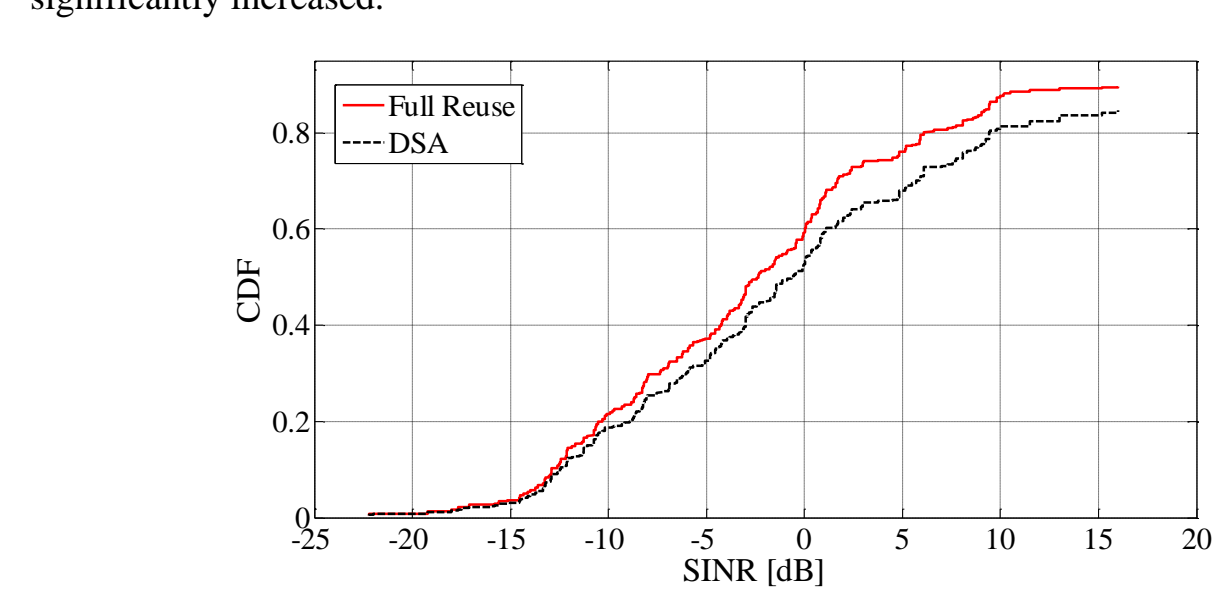

Figure 3. The CDF Curves of SINRs of UEs

However, the blind pursuit of maximum cell throughput will result in less fairness. Therefore, ignoring fairness, it is infeasible to raise the cell throughput by adopting ABS scheme blindly. We proposed the DSAGF to increase cell throughput while guaranteeing fairness. 


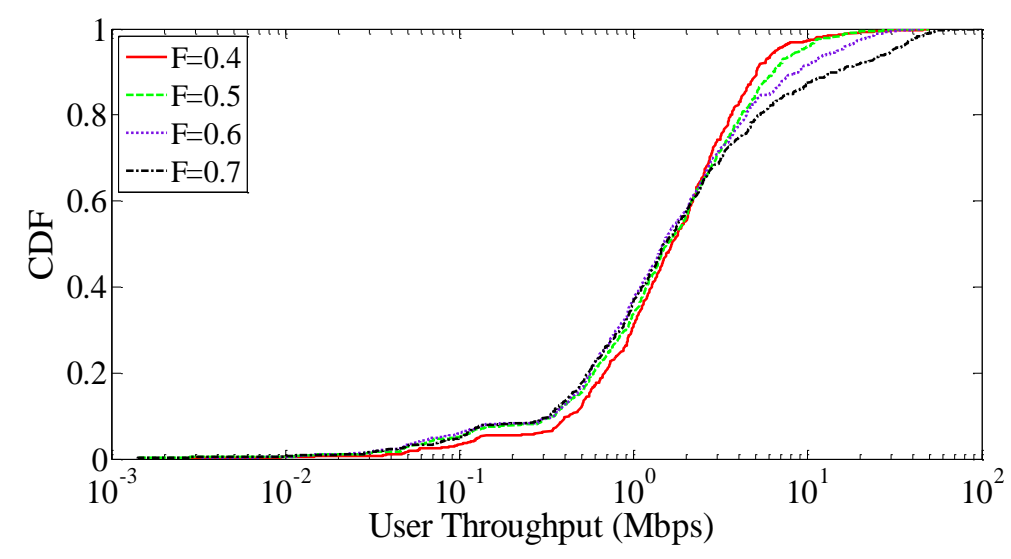

Figure 4. The CDF Curves of User's Throughput with Different Fairness Index

As shown in Fig. 4, the CDF curves of user's throughput are the sharpest when the fairness index is 0.4 , which shows high fairness and small difference of users throughput. With fairness index $F$ gradually increasing, the situation of faimess gets orsening and the difference of throughput among users become biggen

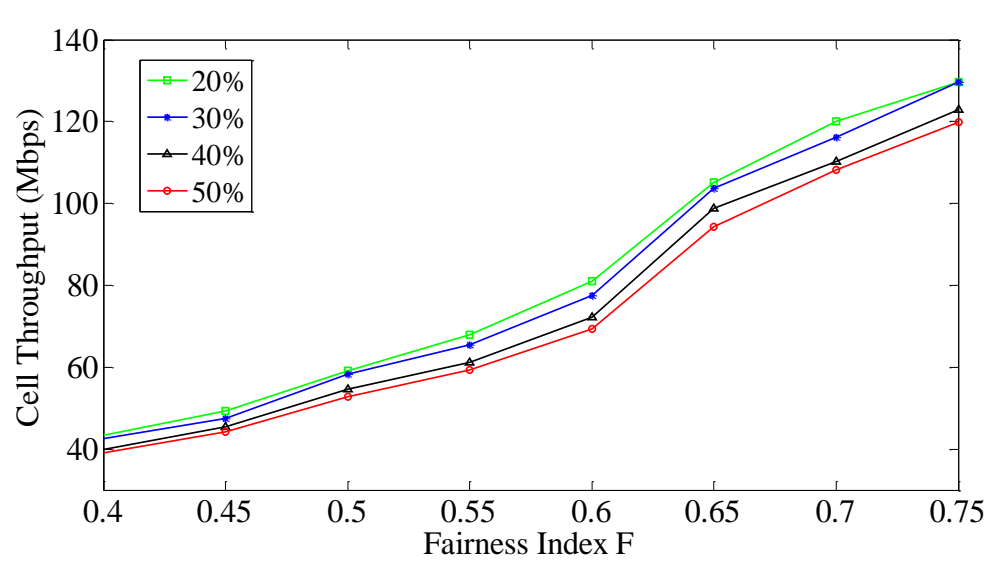

Figure 5. Cell Throughput of User with Different Fairness Index

Fig. 5 illustrates the relation between fairness and cell throughput. The higher proportion of $A B S$ subframes, the fewer subframes capable of transmitting data. We can conclude that cell throughput declines when the proportion of ABS subframes increases. It means that the blind pursuit of maximum cell throughput leads to fairness decrease. Therefore, we should seek a compromise between fairness and cell throughput. 


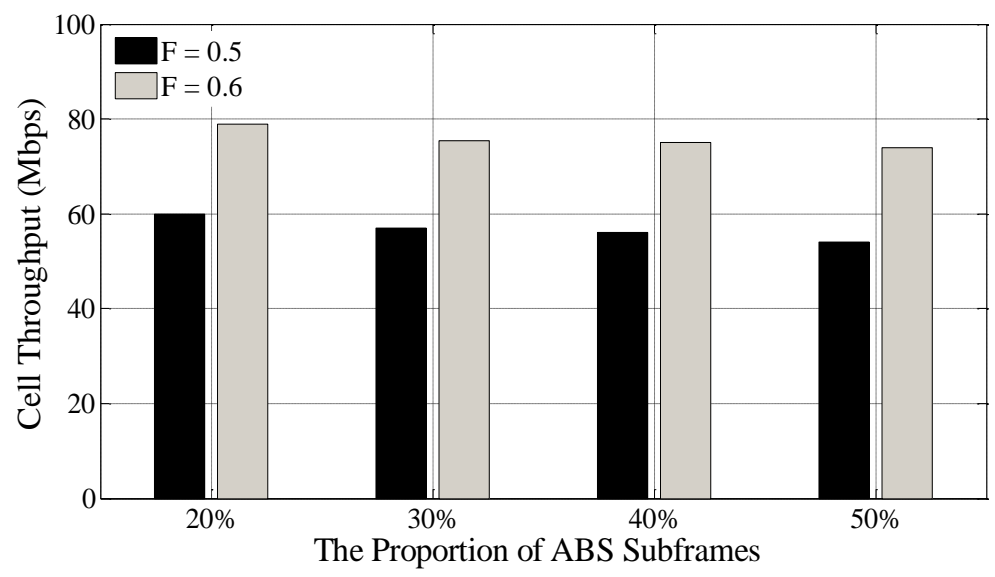

Figure 6. The Cell Throughput with the Proportion of ABS Subframes from $20 \%$ to $50 \%$

As shown in Fig. 6, when the fairness index varies from 0.5 to 0.6 , the sacrifice of fairness contributes to higher throughput. The throughput and Bit Error Rate (BER) of the users are two important performance indexes. Fig. 7 shows the BER of users in HetNets without and with eICIC. In this figure, the horizontal axis is about the change of time and the vertical axis shows that of BERs. Fig. 8 shows the throughput of users in HetNets without and with eICIC. The horizontal axis reveals the change of time and the vertical axis shows that of aser throughputs.

In the situation without eICIC, the communication rates of both small cell UE 1 and macro cell UE 2 are about $450 \mathrm{Kbps}$. However, the BER of UEs is as high as 30-40\% due to the inter-cell interference. Therefore, UEs cannot communicate properly. When the eICIC is used at about the $10^{\text {th }}$ second, user BER drops significantly. At the same time, the throughput of users decreases slightly from that without eICIC to about $300 \mathrm{~kb}$ s because there are some subframes carrying no data. The slight decline of throughput of the users causes the obvious decrease of BER. The throughput of the users decline slightly and the BER decreases obviously, in return.

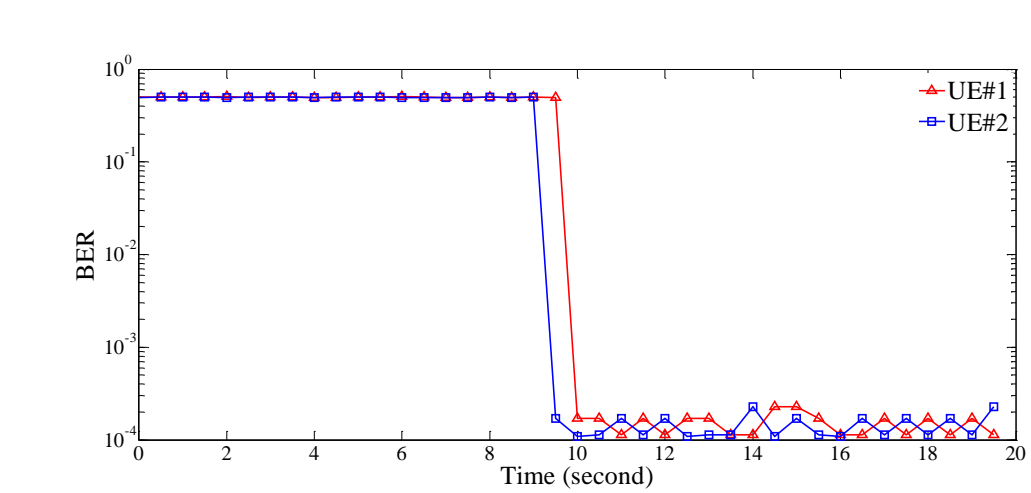

Figure 7. The Contrast of BERs without and with eICIC 


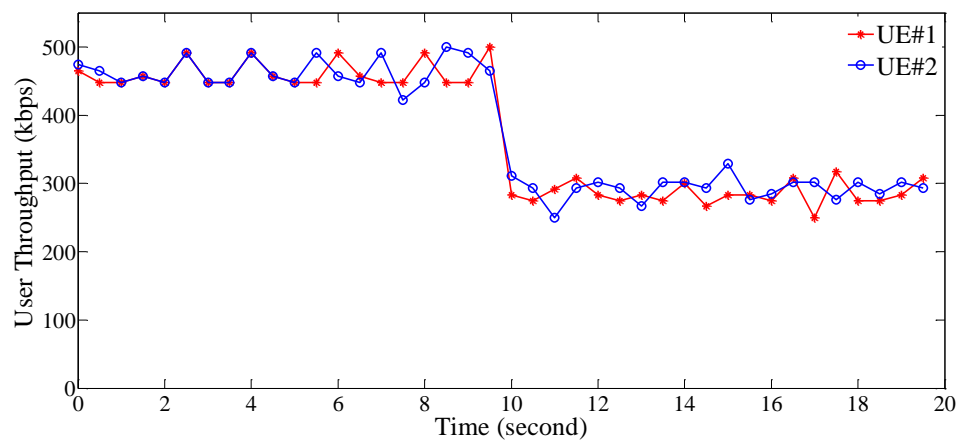

Figure 8. The Contrast of User Throughputs without and with elCIC

\section{Conclusions}

In this paper, the main contribution is to protect control channels particularly for dense and uncoordinated networks. Within this goal, we introduce a dynanic time domain interference mitigation scheme based on ABSs. Simulation results demonstrate that significant improvement has been made in the outage ratios of femto-UEs and macro-UEs by adopting the scheme we proposed. In addition the scheme requires minor extensions in existing LTE signaling procedures. In the scheme, subfranes can be assigned autonomously by BSs, and the BSs are capable of dynamically adapting to the interfering environment. Therefore, we can apply the proposed scheme to unplanned wireless networks without central controller. The flexible assignment of subframes depends on the interference conditions. At last, this improves the untilization of the resources resulting in a high system capacity.

\section{Acknowledgements}

We would like to thank the anonymous teferees whose comments helped us to improve the presentation of thi paper. This work is supported by the Scientific and Technological Research Program of Chongqing Municipal Education Commission (Grant No. KJ1501107, KJ1501105), and the Scientific Research Program of Chongqing University of Arts and Sciences (Gran Do. Y2015DQ35, Z2015DQ10, Z2015DQ08).

\section{References}

[1] Cisco Visual Networking Index, "Global mobile data traffic forecast update, 2013-2018", white paper, CISCO Inc., (2014)

[2] China Mobile Research Institute, "C-RAN the road towards green RAN", white paper, China Mobile Communications Corporation Inc., (2011)

[3] A. Ghosh, R. Ratasuk, B. Mondal, N. Mangalvedhe and T. Thomas, "LTE-Advanced: next-generation vireless broadband technology", IEEE Wireless Commun., vol. 17, no. 3, (2010), pp. 10-22.

[4] A. S. Hamza, S. S. Khalifa, H. S. Hamza and K. Elsayed, "A survey on inter-cell interference Coordination techniques in OFDMA-based cellular networks", IEEE Communications \& Tutorials, vol. 15, no. 4, (2013), pp. 1642-1670.

[5] A. Damnjanovic, J. Montojo, Y. B. Wei, T. F. Ji, T. Luo and M. Vajapeyam, "A survey on 3GPP heterogeneous networks", IEEE Wireless Commun., vol. 18, no. 3, (2011), pp. 10-21.

[6] D. Lopez-Perez, İ. Güvenc, G. Roche, M. Kountouris, T. Q. S. Quek and J. Zhang, "Enhanced intercell interference coordination challenges in heterogeneous networks", IEEE Wireless Commun., vol. 18, no. 3, (2011), pp. 22-30.

[7] M. Simsek, M. Bennis and I. Güvenç, "Learning based frequency and time-domain inter-cell interference coordination in HetNets", IEEE Transactions on Vehicular Technology, vol. 64, no. 10, (2015), pp. 4589-4602.

[8] TS 36.211, Evolved universal terrestrial radio access (E-UTRA); physical Channels and Modulation, tech.rep., 3GPP, (2013) 
[9] TS 36.300, Evolved universal terrestrial radio access (E-UTRA) and evolved universal terrestrial radio access network (E-UTRAN); overall description; stage 2, tech.rep., 3GPP, (2013)

[10] R1-104968, Summary of the description of candidate eICIC solutions, tech.rep., 3GPP, (2010)

[11] N. Sharma and A. S. Madhukumar, "Genetic algorithm aided proportional fair resource allocation in multicast OFDM systems", IEEE Transactions on Broadcasting, vol. 61, no. 1, (2015), pp. 16-29.

[12] D. Parruca, M. Grysla, S. Görtzen and J. Gross, "Analytical Model of proportional fair scheduling in interference-limited OFDMA/LTE networks", Proceedings of the 78th IEEE Vehicular Technology Conference, Las Vegas, USA, (2013) September 2-5.

[13] M. I. Kamel and M. F. K. Elsayed, "Performance evaluation of a coordinated time-domain eICIC framework based on ABSF in heterogeneous LTE-Advanced networks", Proceedings of the IEEE Global Communications Conference, Anaheim, USA, (2012) December 3-7.

[14] R1-130754, System simulation results on heterogeneous networks with cell range extension, tech.rep., Renesas Mobile Europe Ltd, (2013)

[15] S. N. S. Kshatriya, S. Kaimalettu, S. R. Yerrapareddy, K. Milleth and N. Akhtar, "On interference management based on subframe blanking in heterogeneous LTE networks", Proceedings of the 5th International Conference on Communication Systems and Networks, Bangalore, India, (2013) January 7- 10 .

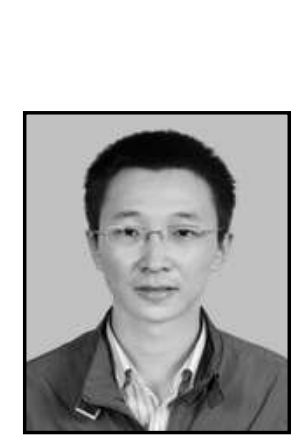

\section{Authors}

Peng Li received his Bachelor's degree in Electronic Science and Technology from Sichuan University Chengdu, in 2004. He received his Master's degree in Communication and Information System from Chongqing University, Chongqing in 2010. He has been studying Communication and Information System in College of Communication Engineefing, Chongeing University, Chongqing, since 2012. He has actively contributed to design and implementation of LTE, LTE-Advanced physicalaryer and MAC layer by software defined radio. He is researehing SDR, Cognitive Radio and LTEAdvanced HetNet. Since 2010 he has been working as a lecturer in School of Electronic and Electrical Engineering, Chongqing University ot Arts and Sciences, Chongqing, China.

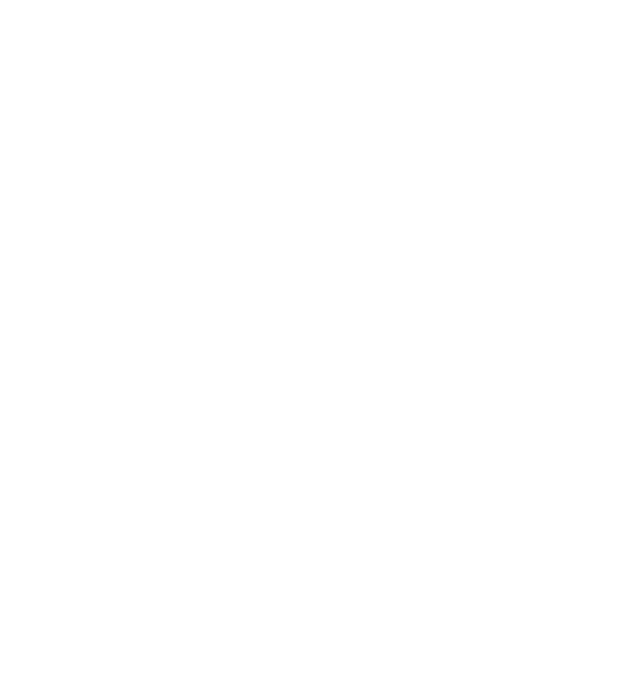

